Running head: PURITY NORMS AND ENVIRONMENTAL BEHAVIOR

An Integrative Framework to Test Purity Motivations in Environmental Behavior

\author{
Sonya Sachdeva \\ Northern Research Station, U.S. Forest Service \\ Reihane Boghrati \\ Department of Computer Science, University of Southern California
}

Morteza Dehghani

Department of Psychology \& Computer Science, Brain and Creativity Institute, University of Southern California

\begin{abstract}
Author Note
Correspondence concerning this article should be addressed to Sonya Sachdeva, USDA Forest
\end{abstract}

Service, Northern Research Station, Evanston, IL, sonyasachdeva@fs.fed.us. 


\begin{abstract}
Environmental issues are often discussed in purity-related terms. For instance, pollution, contamination, toxicity, and degradation are all concepts that can evoke notions of (im)purity in an environmental context. In this paper, we assess the efficacy of purity-based norms as drivers of environmental behavior. First, using a social media-based environmental cleanup campaign as a test case, we find that purity based norms, increase participation in the campaign. We then replicate and extend these findings in three behavioral experiments, finding that the effects of purity on environmental behavior are strongest for people who are more deeply connected with an in-group. Using an integrative approach to combine computational linguistics with behavioral experiments, we find that purity-based norms can be powerful motivators of environmental behavior, particularly if they emphasize the relation to group identity.
\end{abstract}

Keywords: moral psychology; purity norms; environmental behavior; computerized text analysis; behavioral experiments, developing nations 


\section{An Integrative Framework to Test Purity Motivations in Environmental Behavior}

As environmental pressures and natural resource scarcity intensify in the post-industrial era, many activists, not-for-profits, governments, and other large-scale institutions are looking for ways to promote more individual-level pro-environmental behavior. Research over the past several decades has revealed that there are numerous pathways to environmental behavior and engagement - for instance, one's peer group, social incentive structures, product design, personal religious beliefs, have all been shown to be impactful (Sachdeva, Jordan \& Mazar, 2015; Sachdeva, 2016a). However, the most successful interventions and pathways to promoting environmentally-friendly behavior might need alignment between policies and individual attitudes and beliefs. For many people, the decision to make environmentally-friendly choices comes down to moral norms and values, and it is likely that successful appeals designed to promote environmental behavior will need to take values into account. Purity may be one such value and may play an important role in shaping environmental attitudes.

Among the pantheon of moral values, identified by Richard Shweder, Jonathan Haidt, and others (Haidt, Koller, Dias, 1993; Haidt, 2007; Shweder, Much, Mahapatra, 1997), purity concerns may hold special power. While moral philosophers and psychologists typically focused on the moral values of harm and equality as the foundations of human moral judgment, purity concerns have been key in upholding societal and moral order in many small-scale societies (Douglas, 1966). In recent years, violations of purity have been shown to be just as meaningful as violations of harm, and even more so among certain cultural, economic and ideological groups within industrialized countries (Haidt, Koller \& Dias, 1993; Haidt, Graham, Nosek, 2009; Haidt \& Graham, 2007). For instance, poor and working-class communities in major American urban centers appear to judge purity violations as harshly as those involving harm or injustice (Haidt, Koller \& Dias, 1993). Moreover, within conservative political groups in the US and abroad, purity-based violations carry more weight than among liberals (Haidt \& Graham, 2007). In India, within urban and rural communities, purity concerns are the basis of moral judgments, with strict implicit and explicit codes to preserve moral and spiritual purity (Shweder, Much, Mahapatra, 1997). From an 
evolutionary perspective, purity as a moral domain may have evolved from purely biological concerns, such as cultural guidelines designed to prevent exposure to potentially life-threatening toxins and pathogens (Neusner, 1975; Rozin, 1996; Sosis \& Alcorta, 2003; Atran \& Norenzayan, 2004; Boyer, 2008) which eventually extended to the moral domain, preserving purity in one's actions, intentions and spirit.

Furthermore, purity concerns may apply to the environmental domain as well. The natural world is often described in the terminology of purity, often evoking ideas of pristine, untouched landscapes (Bernbaum, 2006; Gadgil \& Vartak, 1976; Harris et al., 1966; Lebbie \& Guries, 1995; Sosis, 2011). Environmental pollution can be thought of as defiling, contaminating, or adulterating shared resources such as air or water. There is also some evidence to suggest that purity-based notions of the environment can be beneficial for conservation behavior. Children as young as seven are more likely to engage in environmental protection if they believe that doing so would preserve the purity of the natural world (Rottman \& Kelemen, 2012). People with more conservative political viewpoints are more likely to respond to purity-based environmental appeals, e.g., more disgust-inducing images, than harm-based ones (Feinberg \& Willer, 2012). And for Hindu-Indians, the perceived purity of a natural resource also appears to be an important factor in how polluted they perceive that resource to be (Sachdeva, 2017).

How purity exerts its influence in the environmental domain is yet unclear. One explanation is that biocentrism, care for the natural world beyond its impact on humans, has at least two distinct components that mirror the harm versus purity delineation in moral reasoning. As Rottman \& Kelemen (2012) propose, harm-based biocentrism may operate by extending the moral circle to natural kinds perceived to have minds and sentience. However, purity-based biocentrism may be distinct in its more communal or systemic view of the natural world. If we assume that one component of environmental protection necessarily entails a tradeoff between short-term, individual consumption and long-term collectively beneficial outcomes (Karp, 1996; Hardin, 1968), a purity-based environmental concern may allow for a temporary bridging between these divergent incentives. As research has shown, purity, more than other types of moral concerns, can create and bind social communities (Graham \& Haidt, 2010). For instance, people who hold similar ideas about contamination and cleanliness (as opposed to ideas about 
harm) are more willing to share spaces and have been shown to have closer social ties (Dehghani et al. 2016). Perhaps purity, as a binding or group-focused moral value, can be essential in unifying spheres of human-nature existence where harm-based morality may even serve to reify these boundaries.

Therefore, our aim in this project was to understand whether purity concerns motivated proenvironmental behavior and in particular, if this relationship was moderated by in-group identity. Furthermore, unlike previous studies assessing the impact of purity concerns on environmental values and behavior, we tested these ideas with Indian populations, a country where purity is of paramount importance within the moral domain (Jensen, 1998). We employed a mixed-methodological approach, combining social media analyses with confirmatory online experiments to understand the relationship between purity and environmental behavior. First, we tested our ideas in the naturalistic context of the 'Clean India campaign,' a social media based initiative launched within India to promote sanitation, recycling, and general environmental awareness, as well as curbing littering and other polluting practices. This campaign allowed us to assess the real-world impact of purity and in-group based appeals on environmental attitudes and behaviors. We then further tested our hypotheses with a series of preregistered experiments to understand the causal impact of purity-based appeals on environmental behavior and whether the effect is moderated by in-group concern and connection.

\section{Study 1}

This study assessed the impact of the Clean India campaign, implemented by the Indian government in the fall of 2014. Specifically, we were interested in assessing changes in social media rhetoric and behavior surrounding environmental issues. The Clean India campaign, launched on the anniversary of Mahatma Gandhi's birth, was wide-ranging in scope but its main objectives included the elimination of open defecation, promoting behavioral change with regard to sanitation practices and increasing awareness of environmental issues such as clean air and water (Jeffrey, 2015; "Swachh Bharat", 2014). The campaign, similar to other challenge-based campaigns, such as the ALS Ice Bucket challenge ("Ice Bucket Challenge", n.d.), itself was experienced by most people on social media sites such as Twitter and 
Facebook where users could discuss the campaign, their behavior, as well as nominate others in their social networks and beyond to participate.

The Clean India campaign provided a unique research opportunity as a platform for testing the role of purity-based appeals. Not only was it primarily focused on cleanliness and removing the stigma of India as dirty or unclean (Jeffrey, 2015; Doron, 2016; Gupta, Coffey \& Spears, 2016), the campaign actively promoted widespread civic action in service of restoring the purity of India as a nation (Doron, 2016). Many, if not most, of the promotional materials for the campaign referenced Mahatma Gandhi, as the Father of the Nation, once again reinforcing the patriotism inherent in participating in the campaign. Therefore, not only did the campaign highlight purity as a goal to be achieved through the campaign, but it also relied on a shared group identity to meet that goal.

Because the campaign was primarily promoted on social media sites, we used conversations on Twitter before and after the onset of the campaign to test its effectiveness. We probed Twitter conversations for two dimensions of effectiveness: 1) whether it was effective in increasing awareness of environmental issues and 2) its efficacy in increasing green or environmentally-friendly behavior.

\section{Method}

We first compiled a set of hashtags related to the Clean India campaign by relying on official Indian websites that listed these hashtags (see Supplementary Material for the list of the hashtags). We then purchased tweets that included any of these hashtags and were posted between September 2014 (before the start of the campaign) and December 2014 from Gnip.com. This resulted in 6,735,128 tweets.

To prepare tweets for text analysis, we applied a series of preprocessing steps on the dataset. Preprocessing and cleaning the data helps to discard unnecessary information and increase text analysis accuracy. First, we replaced every web page address written as part of the tweets with "URL", and every user mentions with "AT_USER". This is an essential step because specific web page addresses or and user references could alter the result of text analysis. Next, we removed redundant symbols from the beginning and end of tweets. Also, we removed all extra white spaces. Finally, we detected elongated 
words, which are defined as words with more than three consecutive occurrences of the same character. We handled these words by removing the extra characters.

After preprocessing the tweets, we used topic modeling to explore the various themes discussed during the discussion of the campaign. Topic modeling is a frequently used text analysis approach which aims to discover underlying topics discussed in a set of documents (Iliev, Dehghani \& Sagi, 2015). We decided to use a fully data-driven, bottom-up approach, rather than using the Moral Foundations Dictionary (Graham, Haidt \& Nosek, 2008) or any derivative of it (Garten et al., 2017), because our corpus is a mix of English and Hindi (written in Latin script) tweets, and any dictionary-based approach would not have fully captured the constructs of interest in this situation. A general assumption by topic modeling approaches is that a document is represented as a distribution of a mixture of several different topics. A very well-known topic modeling algorithm is Latent Dirichlet Allocation (LDA; Blei, Ng \& Jordan, 2003). LDA is a generative statistical model which aims to find a mixture of topics and their proportion for each document. In other words, LDA calculates the probability of each topic for each document in the corpus. Topics are represented as a probability distribution over words in the vocabulary (all the words available in the corpus). The probability of words occurring in a topic provides a general idea of the content of the topic.

In LDA, the number of topics is manually set. With a larger number of topics, LDA discovers more fine-grained topics, while if the number of topics is small, LDA extracts more general topics. In our analysis, we ran LDA with 10, 40, 100 and 200 topics. The general trend of topics did not vary greatly, but we decided to go with 100 as it provided the most coherent set of topics.

By using LDA we were able to assess: 1) The composition of topics discussed in our corpus and 2) The likelihood or probability of each topic being discussed in a given tweet. To assess which of the 100 topics were about the campaign, we first did a weighted word count of the top 20 words for each topic (words with the highest probability in each topic), using a set of Clean India related keywords (see Supplementary Materials for details). Specifically, we summed the probabilities of Clean India related keywords to assess the overall probability that a topic was about the campaign. These keywords only 
occurred in 8 (out of 100) of the topics. Three topics with the highest probability of campaign-related words were chosen as the target topics. The first topic (Topic 20) had a probability of 0.42 of being related to the Clean India campaign. The second (Topic 67) had a probability of .33, and the third highest probability (Topic 7) was 0.19 (see Supplementary Materials for other topics weights). The topics with the highest probability of being related to the campaign were also highly stable across the different topic models tested. We did not seek IRB approval for this study.

\section{Results}

A preliminary assessment of the three topics most likely to be related to the Clean India campaign suggested that the topics differed in how people were discussing the campaign. The first (Topic 20) and third (Topic 7) Clean India related topics were composed of words such as 'initiate', 'launch', 'request' and 'effort'. These topics reflect the more behavioral aspect of the campaign where participants could nominate others in their social network to participate in cleanup activities or post pictures of themselves engaging in anti-littering campaigns and other activities. The second topic (Topic 67), on the other hand, composed of words such as 'pollution, waste, toilet', appeared to be a more descriptive topic about general environmental and sanitation issues the campaign was designed to address.

To verify these initial observations we recruited 51 independent Indian raters from Amazon's Mechanical Turk, blind to our initial assessment and hypothesis. These raters were asked to read through lists of words comprised of the 20 most frequent words from each topic, excluding words directly related to the Clean India campaign (e.g., Clean, campaign, swacch, bharat, abhiyaan). Each participant was asked to rate each word, on a four-point scale, on how likely it was that the word could be used to describe an action or behavior.

A reliability analysis on each topic's 20 most frequent words showed high internal reliability within the topics (see Supplementary Materials for details), providing a human validation of the semantic coherence of the Topic model that was generated. We then calculated the average for each topic's words on the participants' behavior ratings. On a four-point scale with four indicating a word most likely to 
refer to a behavior, the mean action/behavior rating for topic 7 was $3.17(\mathrm{SD}=.52)$. The mean behavior rating for topic 20 was $3.09(\mathrm{SD}=.38)$ and for topic 67 was $2.97(\mathrm{SD}=.66)$. To assess whether these topics were statistically different from each other on behavior ratings, we conducted three paired-samples t-tests. These showed that while topic 7 and topic 20 were not significantly different from one another $(\mathrm{t}(44)=1.87, \mathrm{p}=.07)$, both were rated as more behavior-related than topic 67 (topic 7 vs. 67, $\mathrm{t}(47)=$ $3.01, p<.001$; topic 20 vs. $67, \mathrm{t}(44)=2.41, \mathrm{p}=.02)$. These analyses suggested that topics 7 and 20 were both related to the behavioral aspects of the Clean India campaign whereas topic 67 reflected some other dimension, perhaps a general description of the issue. Thus, for the subsequent analyses, topics 7 and 20 were collapsed (i.e., their weights averaged) into a single behavioral topic and contrasted with topic 67. We then divided the tweets into two time periods to assess change over time in the target topics the topic containing the behavioral aspect of the Clean India campaign and the other campaign-related prevalent topic. Timeframe 1 was the initial period of the campaign, including a few days before the launch (September 30th - October 8th, 2014) to inform a baseline of conversation about the campaign. Timeframe 2 included the period after the campaign had been implemented for a few weeks (November 19th through December 8th, 2014). To assess the change in the prevalence of the topics during the two time-periods, we performed a $2 * 2$ ANOVA with timeframe as the first factor, topic category as the second, and topic weight as the dependent variable (Figure 1). The main effects of time and category were negligible (Time: $\eta_{\text {partial }}{ }^{2}=6.52 * 10^{-5}$; Topic category: $\eta_{\text {partial }}{ }^{2}=1.83 * 10^{-5}$ ). The interaction between the two factors resulted in an effect size of $0.013^{1}(\mathrm{~F}(1,6087480)=7664.0, p<0.001)$. In Timeframe 1 , the

\footnotetext{
${ }^{1}$ To quantify these effect sizes in the context of topic interactions in our dataset, we calculated all possible interactions between all the 100 topics in addition to the averaged behavior topic discussed above. This resulted in $50502 * 2$ interactions. We then investigated where the effect size of the interaction of the constructs we are interested in fell within the realm of all of the effect sizes of the $2 * 2$ interactions between the LDA topics. The effect sizes of the 5050 interactions formed a long-tail distribution with the above interaction effect size (0.013) falling in the $80 \%$ quantile.
} 
two target topics' prevalence differed dramatically across tweets, with the behavior related topics $(\mathrm{M}=$ $0.0095, \mathrm{SD}=0.0051)$ being discussed less frequently than the other prevalent campaign topic $(\mathrm{M}=0.010$, $\mathrm{SD}=0.0081)$ (See Table 1 for the all the comparisons). In Timeframe 2, several weeks after the launch of the campaign, the behavioral topic reached an elevated level of prevalence compared to the start of the campaign $(\mathrm{M}=0.0101, \mathrm{SD}=0.0082)$, while the other topic decreased in prevalence $(\mathrm{M}=0.0096, \mathrm{SD}=$ $0.0077)$.

Table 1. Comparisons of different time points for the two topics of interest

\begin{tabular}{|c|c|c|c|c|c|}
\hline Comparison & $99 \%$ CI & $d$ & $d f$ & $t$ & Sig. \\
\hline $\begin{array}{c}\text { Behavior at Time 1 vs. } \\
\text { Behavior at Time 2 }\end{array}$ & {$[-.0007,-.0006]$} & 0.0975 & 2040200 & -78.962 & $<.001$ \\
\hline $\begin{array}{c}\text { Other topic at Time 1 vs. } \\
\text { Other topic at Time 2 }\end{array}$ & {$[0.0003,0.0004]$} & 0.0509 & 2879900 & 44.276 & $<.001$ \\
\hline $\begin{array}{c}\text { Behavior at Time 1 vs. } \\
\text { Other at Time 1 }\end{array}$ & {$[-0.0005,-0.0004]$} & 0.0752 & 2929700 & -70.226 & $<.001$ \\
\hline Behavior at Time 2 vs. & {$[0.0005,0.0006]$} & 0.0676 & 2594400 & 54.536 & $<.001$ \\
Other at Time 2 & & & & & \\
\hline
\end{tabular}




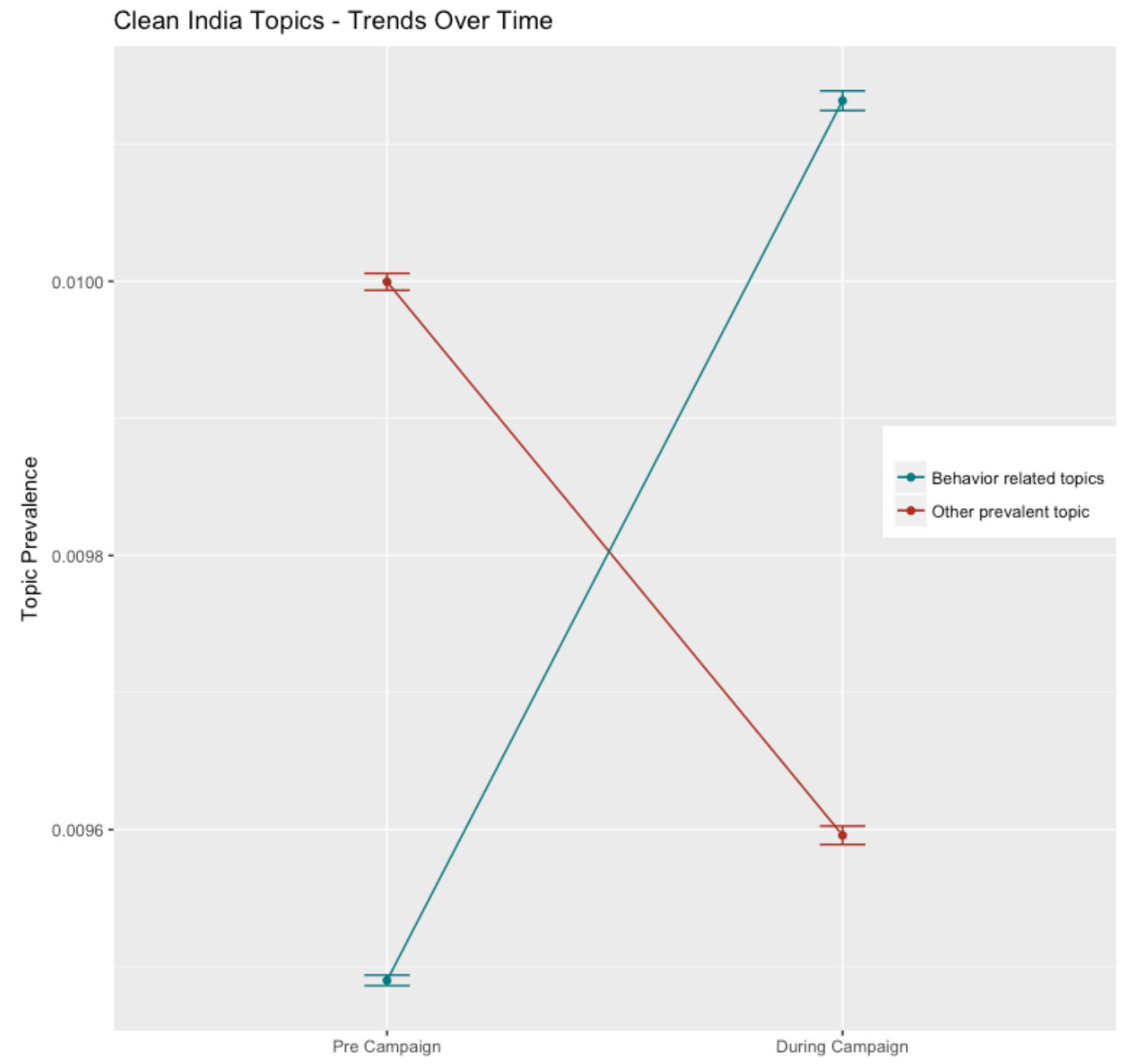

Figure 1: Change over time in the topic containing the behavioral aspect of the Clean India campaign and the other campaign-related prevalent topic.

\section{Discussion}

The onset of the Clean India campaign provided a unique research opportunity to test the impact of a large-scale environmental campaign. The campaign was strongly rooted in cultural ideals of purity while also employing patriotic imagery of national figures, such as Mahatma Gandhi. This study measured the impact of the campaign over a two-month period in increasing environmental awareness and behavior. Because the campaign was heavily marketed on social media sites, we used conversation on Twitter as 
proxies for the environmental issues the campaign was designed to address, as well as to measure behavioral engagement with the campaign, e.g., participation in cleanup activities.

Trends over the eight-week period revealed that behavioral engagement with the campaign increased relative to baseline levels, even as other Clean India related topics showed a decline in the same time period. These results suggested that the purity and in-group focus of the Clean India campaign were effective drivers of environmental behavior, particularly as there was no sustained effects with discussion of the other sanitation related issues of the campaign.

This study provided initial confirmation of the hypothesis that a campaign utilizing purity and patriotism in its appeals could increase environmental behavior. However, it is unclear whether these appeals based on purity and national pride could be more powerful than other ecocentric or anthropocentric environmental appeals, for instance. It is also unclear which aspect of the Clean India campaign was most likely to yield these positive environmental impacts - are purity concerns or elements of national pride sufficient or as we posit, does the confluence of purity and in-group salience foster greater environmental behavior? These ideas were further tested, within experimental contexts, in the next three behavioral studies.

\section{Study 2}

The results from Study 1 suggest that purity and group-based appeals in the context of the Clean India campaign increased the prevalence of behavior related tweets on social media. In Study 2, we tested whether appeals designed with the same structure as those used in the Clean India Campaign might increase intentions to engage in green behavior in an experimental setting.

\section{Method}

Participants $(\mathrm{n}=262)$ were recruited from Amazon Mechanical Turk, an online crowdsourcing platform. To determine the sample size, we conducted a power analysis using an estimated effect size of .25, which suggested a required 252 participants to achieve a power level of .90 . Additional participants were recruited to compensate for attrition and incomplete responses. 
We restricted the sample to participants from India to maintain some degree of cultural consistency from the first study. Of this sample, 51 participants were removed due to participant attrition and incomplete surveys leaving a final sample size of 211 participants $(34.6 \%$ female, mean age $=32.45$ years, $\mathrm{SD}=9.43$ ).

Each participant was randomly assigned to receive either a purity-based or ecocentric appeal to engage in environmental conservation. The purity-based text was adapted from language that was used during the Clean India campaign while the ecocentric text was adapted from speeches given by a prominent environmental activist within India. Both texts were validated using an independent sample of Mechanical Turk workers $(\mathrm{n}=26)$. These analyses showed that the text adapted from the Clean India campaign was indeed more reflective of notions of purity while the ecocentric text elicited notions of the natural world and all living beings within it (See Supplemental Materials for further information on the validation methodology).

After reading either the purity-based or ecocentric appeal, participants were asked to judge each text on ease of comprehension and effectiveness of the message. The inclusion of these questions achieved two objectives: 1) Reading the text with these questions in mind helped create a deeper engagement in the task, ensuring that the messages were more deeply encoded in the minds of the participants and 2) Participants' responses to the ease and effectiveness questions could subsequently be used as covariates in the analytic model, to control for individual-level variation in text comprehension. Willingness to engage in green behavior was assessed by asking participants their subjective probability of engaging in seven distinct individual and household level conservation behaviors. These included turning lights off when not in use, making recycling a priority and eating vegetarian meals more often (refer to Supplementary Materials for complete list). For each behavior, participants indicated their likelihood of engaging in the action on a 5 point scale. Finally, we measured participants' baseline environmental concern using the New Ecological Paradigm (Dunlap et al. 2000) and included a set of demographic questions, measuring participants' education level, political orientation, religiosity, gender, and age. This study was approved by the USC IRB panel (UP-15-00380). 


\section{Results}

A reliability analysis done on the seven green behavioral items showed that participants' willingness to engage in these behaviors was internally consistent (Cronbach's $\alpha=.77)$. The items were subsequently collapsed into an overall index of willingness to engage in green behavior. Because most participants were quite willing to engage in a majority of the green behaviors listed in our study, this variable was highly left-skewed. Therefore, we split the index into quartiles for further analyses.

A Generalized Linear Model was fit to the data to test the impact of the experimental condition, i.e., participants reading either the purity-based or ecocentric environmental appeal, on willingness to engage in green behavior, controlling for baseline environmental concern, demographic variables and text comprehension. This analysis revealed that the type of environmental appeal participants read had a significant effect on reported willingness to engage in green behavior $(\beta=.33, \mathrm{t}(201)=2.25, \mathrm{p}=.03$, $\eta_{\text {partial }}{ }^{2}=.02$ ), controlling for all covariates reported above. Participants who read the purity based appeal were willing to engage in more green behaviors $(\mathrm{M}=2.42, \mathrm{SD}=1.10)$ than participants who read the ecocentric appeal $(\mathrm{M}=2.17, \mathrm{SD}=1.08)$, providing partial support for our central hypothesis. Among the covariates tested in the model, age $\left(\beta=.02, \mathrm{t}(201)=2.00, \mathrm{p}=.047, \eta_{\text {partial }}{ }^{2}=.02\right)$ and gender $(\beta=.31$, $\left.\mathrm{t}(201)=2.00, \mathrm{p}=.047, \eta_{\text {partial }}{ }^{2}=.02\right)$ affected participants' willingness to engage in green behaviors. Interestingly, neither participants' baseline environmental concern, as measured by the New Ecological Paradigm, nor their political orientation, had any bearing on expressed green behavior willingness. Of the text characteristic questions, ease of comprehension had no impact but perceived effectiveness of the text appeared to positively affect willingness to engage in green behavior $(\beta=.23, \mathrm{t}(201)=2.00, p<.047$, $\left.\eta_{\text {partial }}{ }^{2}=.02\right)$.

\section{Discussion}

Study 2 provided an initial confirmation of the results observed in our naturalistic field assessment of the Clean India campaign in a controlled, randomized experiment. As in Study 1, we found that appeals similar to those employed in the Clean India Campaign, were more likely to galvanize environmental action than more ecocentric appeals. As in the actual campaign, there appeals were not designed 
unambiguous tests of a purity-based environmental appeal. Rather, these appeals conflated the effects of in-group salience with notions of environmental purity, yielding a similar effect on environmental behavior in the experimental context of Study 2 as we observed in the naturalistic design of Study 1. We then designed Studies 3 and 4 to tease apart the relative impact of purity and in-group identity in fostering environmental behavior.

\section{Study 3}

Study 3 expanded on the previous study in two important ways. First, this study was designed to test the moderating role of in-group identity in the relationship between purity concerns and environmental behavior. That is, we were interested in learning whether a focus on one's in-group would amplify the effect of purity-based appeals in motivating environmental behavior. Second, the previous study utilized purity and environmental appeals that closely mirrored those used in the Clean India campaign. In Study 3, we primed purity concerns using paradigms that had been previously used by other researchers and therefore had high construct validity.

\section{Method}

Participants $(n=495)$ were recruited from Amazon Mechanical Turk. To determine the sample size, we conducted a power analysis using a conservative estimated effect size of .20, which suggested a required 418 participants to achieve a power level of .90. The sample was limited once again to participants from India. Of these, 87 participants were removed due to subject attrition and incomplete responses (i.e., not completing main independent and dependent measures). This left a final sample of 408 participants $(20.8 \%$ female, mean age $=30.03$ years, $\mathrm{SD}=7.98)$.

Each participant was randomly assigned to either the purity or ecocentric appeal condition. However, in this study, we used a circumscribed purity and ecocentric manipulation that was adapted from Feinberg and Willer (2013). Participants were asked to read passages with embedded images that were either purity-based or ecocentric in content. That is, participants in the purity condition saw pictures of polluted water and were told that the environment is toxic, while participants in the ecocentric condition were shown a picture of a bleached coral reef and read about deforestation, as in the original 
Feinberg \& Willer (2013) study (full materials available at https://osf.io/qd9m2). After reading through these texts, participants were asked to describe what they had read in their own words to ensure that participants were fully attending to the task. Next, they completed an identity fusion measure (Swann et al., 2009; Gomez et al., 2011). Using a series of diagrams with various levels of overlapping circles (in addition to scale responses), participants were asked to report the degree of overlap they felt between themselves and their neighbors and community. It was predicted that participants in the purity condition, who also felt close to their in-group, would be more likely to report engaging in green behavior than those that did not feel connected to their in-group.

The dependent variable of interest in this study was a measure of green behaviors that participants remembered engaging in over the past few years (e.g., picking up litter, eating vegetarian meals, voting for officials who support green policy). The list of 10 behaviors was similar to that used in Study 2(with three additional behaviors, please see Supplementary Materials for details), but rather than asking participants to rate willingness to engage in these green behaviors, we asked them how often they had done them. We modified the dependent variable for two reasons: 1) to get a better approximation of green behavior rather than intentions because as previous work has shown, intentions to engage in green behavior do not necessarily translate to behavior and 2) to generalize our findings and assess whether purity based appeals could affect participants' memory of these behaviors (or at least willingness to say that they had engaged in these behaviors). This sort of recall measure, though not used within the environmental domain, has been utilized in previous studies. Building on the seminal description of the availably heuristic in human judgment and decision-making - the tendency to use what more easily comes to mind to make judgments about past events (Tversky \& Kahneman, 1973) - psychologists have shown that the salience of information can be manipulated within an environmental context. For instance, participants who are led to believe that vigorous exercise is harmful to their health will be more likely to state that they had engaged in less vigorous exercise in the past (Ross, McFarland, Conway \& Zanna, 1983). Therefore, it was posited that if purity based environmental appeals are more effective at priming 
green behavior, then participants exposed to this message would also be better at remembering past occasions of engaging in such behaviors.

Participants also completed a series of demographic questions as before, including a question about their perceived cleanliness relative to their neighbors. This question about perceived cleanliness was necessary to obtain a baseline measure of how pure participants viewed themselves. This study was approved by the USC IRB panel (UP-15-00380).

\section{Results}

A reliability analysis conducted on the ten green recall items showed good internal consistency (Cronbach's $\alpha=.75$ ). A mean index of Green Behavioral Recall was created by averaging participants' responses on all ten items. We then used a Generalized Linear Model to assess the impact of Appeal Type (Purity versus Ecocentric), Identity Fusion and Baseline Perceived Cleanliness on Green Behavioral Recall. Exploratory analyses revealed that the only demographic variable related to the dependent variable was Gender. Therefore, it was included as a covariate in the model. Age, political orientation, and religiosity did not appear to be related to participants' recall of green behaviors.

The final model's results showed a significant main effect of the Appeal Type albeit in the opposite direction as was predicted - overall, the ecocentric environmental appeal seemed to elicit greater recall of green behavior $\left(\beta=-2.90, \mathrm{t}(394)=-2.23, \mathrm{p}=.03, \eta_{\text {partial }}{ }^{2}=.00002\right)$ than the purity-based appeal. However, this main effect was qualified by a statistically significant (and pre-registered) interaction between Appeal Type and Identity Fusion. As shown in Figure 2, participants who were shown the purity-based environmental appeal and had a strongly fused group identity remembered engaging in more green behaviors than those who did not strongly identify with their in-group $(\beta=.76, \mathrm{t}(394)=2.09, \mathrm{p}=$ $\left..04, \eta_{\text {partial }}^{2}=.007\right)$. 


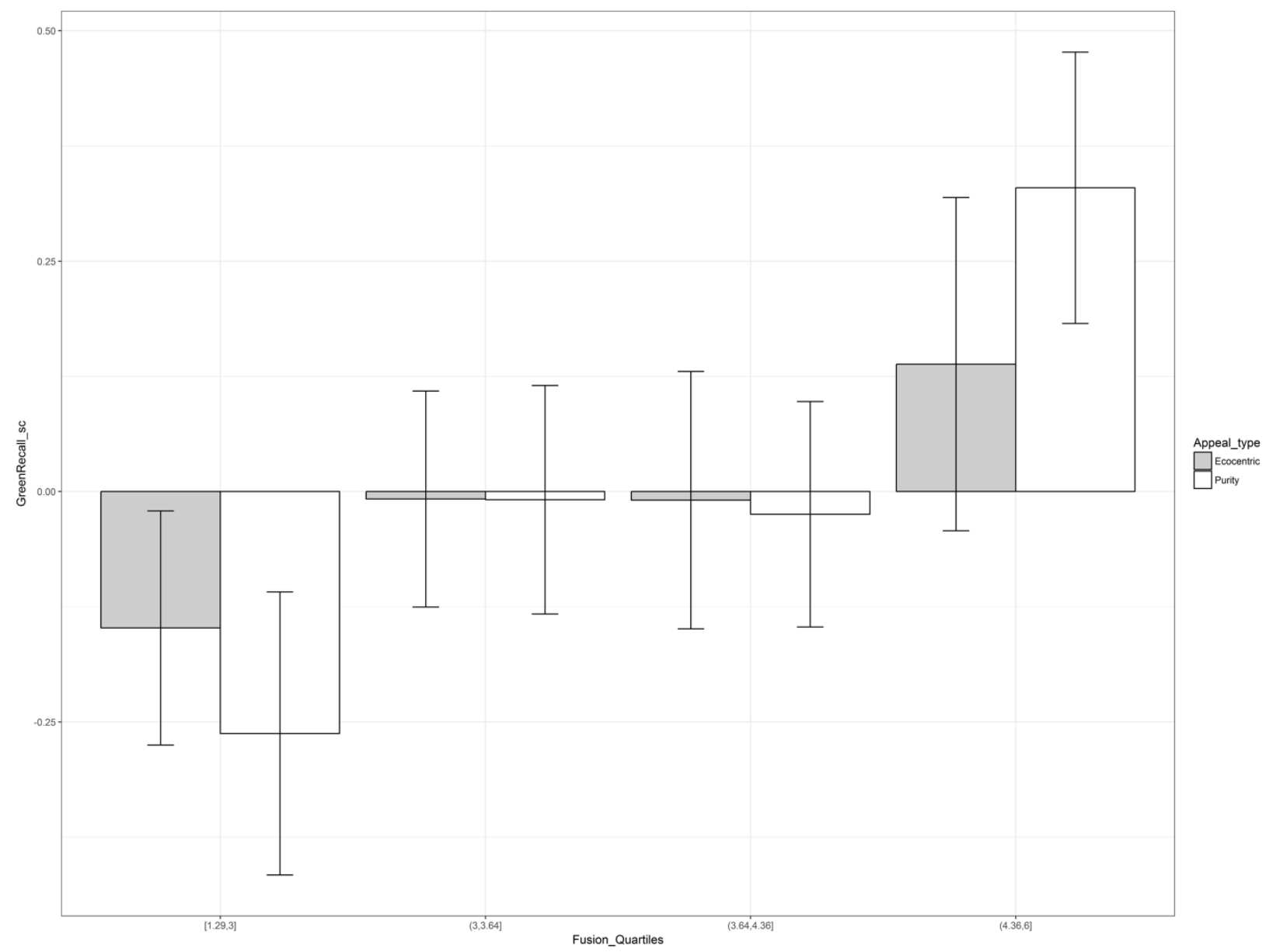

Figure 2: Study 3 results demonstrate that participants who were in the purity-based environmental appeal condition and had a strongly fused group identity remembered engaging in more green behaviors than those who did not strongly identify with their in-group.

\section{Discussion}

In this study, we deepened our understanding of the pathway through which purity-based environmental appeals impact green behavior. The results from Study 3 suggested that purity-based appeals may be powerful motivators of green behavior when they are yoked to a particular in-group. It is also important to note, however, that this study used a recall measure as a proxy for green behavior. Although we cannot claim that the manipulation employed in this study changed past behavior, we believe that the effects on the recall based dependent variable at the very least indicate participants' desire to reduce possible 
dissonance. In other words, in the purity-based condition, participants who were more fused with their ingroup had a stronger belief that they had engaged in environmental behavior. Nevertheless, we addressed this issue by using a more traditional measure of intentional green behavior in the next study.

Furthermore, Study 4 was conducted with the aim to assess the causal impact of group affiliation in moderating the link between purity and green behavior.

\section{Study 4}

Although the previous study provided support for our central argument, that purity, and in-group connection conjointly promote green behavior, we now wanted to test that framework experimentally. Consequently, Study 4 was designed to experimentally manipulate not only the type of appeal participants received in the previous two studies but also how connected they felt to a group. The main hypothesis tested in this study was that green behavior should be highest when participants received the purity appeal and felt most connected to a group. This study was pre-registered on the Open Science Framework (https://osf.io/zg5qx).

\section{Method}

Participants $(\mathrm{n}=1078)$ were recruited from Amazon Mechanical Turk. To determine the sample size, we conducted a power analysis with an estimated effect size of .15, which suggested a required 1009 participants to achieve a power level of .90 . As in the previous two behavioral studies, the sample was limited to participants from India. Of these, 329 participants were removed for not completing the survey (i.e., failing to complete the main experimental primes). Our final sample consisted of 749 participants $(28.6 \%$ female, mean age $=31.05$ years, $\mathrm{SD}=8.18)$.

Participants were randomly assigned to receive the same purity or ecocentric environmental appeal as was used in Study 3. However, before participants saw the environmental appeal, they were asked to complete a sentence unscrambling task (Srull \& Wyer, 1979). This task was framed as a text comprehension task to ensure that participants would be able to complete the remainder of the study. The 
specific materials used in the task were adapted from Zogmaeister et al. (2008) who successfully used them to prime participants with a sense of loyalty or obligation to a group. Participant either had to unscramble ten sentences like "Rahul proved to be loyal in each situation" in the group loyalty condition or "Carla shows a neutral attitude toward the two candidates" in the impartial/equality condition. The ten target sentences were preceded and succeeded by two buffer unscrambling sentences that were common between conditions.

After completing the sentence unscrambling task, participants proceeded to what they were told was the main study where they saw either a purity or ecocentric environmental appeal (same as Study 3), identity fusion measure, and then completed the green behavioral intentions scale. These consisted of behavior such as avoiding plastic bags while shopping, cutting down on garbage produced, and making recycling a priority. Participants were once again given a set of demographic questions, including the question about perceived relative cleanliness used in Study 3. This study was approved by the USC IRB panel (UP-15-00380).

\section{Results and Discussion}

The ten individual items within the Green Behavior measure were once again internally consistent (Cronbach's $\alpha=.84$ ) and were averaged to create the overall measure of Green Behavior. A Generalized Linear Model was once again fit to the data, with the Green Behavior index serving as the dependent variable, and Appeal Type, Group Loyalty/Impartiality, Perceived Cleanliness serving as the independent variables. Identity Fusion was included as a covariate in the model to account for any group affiliation not impacted by the Group Loyalty prime. Finally, age was the only demographic variable shown to affect Green Behavior in exploratory analyses so it, too, was included as a covariate in the model.

Final model results showed a significant main effect of the Appeal Type and a marginally significant effect of the Group Loyalty/Impartiality prime. As in Study 3, the main effect of the appeal suggested that participants who saw the purity-based appeal were less likely to implement green

behaviors in their day-to-day life $\left(\beta=-.78, \mathrm{t}(728)=-2.98, \mathrm{p}=.003, \eta_{\text {partial }}{ }^{2}=.001\right)$ than those who saw the 
ecocentric appeal. And similarly, the marginal main effect of the loyalty prime suggested that participants in the loyalty condition of the sentence unscrambling task were less willing to implement green behaviors $\left(\beta=-.41, \mathrm{t}(728)=-1.90, \mathrm{p}=.06, \eta_{\text {partial }}{ }^{2}=.002\right)$ than those who saw the equality prime. However, critically for our main (pre-registered) hypothesis, the model also revealed a significant positive interaction between Appeal Type and Group Loyalty/Impartiality which suggested that participants who completed the loyalty sentence task and saw the purity-based appeal were most willing to implement green behaviors in their lives $\left(\beta=.83, \mathrm{t}(728)=2.44, \mathrm{p}=.01, \eta_{\text {partial }}{ }^{2}=.009\right)$.

\section{General Discussion}

The present research examined the means through which purity-based moral concerns may motivate environmental behavior. Previous research suggested that for some cultures and ideological groups, purity-based norms may help foster conservation behavior relative to other eco-centric or harm-based norms (Feinberg \& Willer, 2013; Sachdeva, 2017). In this paper, we find that purity-based appeals do appear to positively impact environmental action, but that this effect is moderated by how connected one feels to an in-group. We used a mixed-methods approach in this work, combining insights from a nationally-implemented social media campaign that utilized purity-based norms to increase environmental engagement, i.e., the Clean India campaign, and then complemented this naturalistic assessment with behavioral experiments.

In Study 1, an automated content analysis of Twitter-based discussion of the Clean India campaign found that tweets containing behavioral content, or description of people engaging in cleanup activities related to the campaign increased over an eight week period, even as overall conversation about the campaign decreased. Study 2 elaborated on these results in an experimental setting showing that similar appeals as those used during the Clean India Campaign could increase green behavioral intentions in an online study. Finally, Studies 3 and 4 showed that the positive effect of purity on environmental behavior was moderated by the degree to which participants felt connected to an in-group, either through 
self-reported identity fusion (Study 3) or through priming participants to feel loyalty to an in-group (Study 4).

Interestingly, we found that in the context of other variables tested such as identity fusion or perceived cleanliness of oneself relative to one's in-group, purity-based appeals actually had a negative effect on environmental behavior in Studies 3 and 4. Yet, in conjunction with identity fusion or primed alongside in-group loyalty, purity-based appeals actually became stronger than other ecocentric-focused appeals. These results indicate that purity can be an important pathway towards environmental behavior, independently of political orientation as much of the literature has previously proposed (e.g., Feinberg \& Willer, 2013; Wolsko, Ariceaga \& Seiden, 2016). Rather, purity-based norms become stronger in the context of group relationships and perhaps shift motivations to engage in environmental behavior.

\section{Limitations}

The studies presented here used varied and distinct approximations of green behavior (green behavior recall, intentions, behavior-related tweets), though no actual green behavior (donations to environmental groups, etc.) was measured. The use of green recall measures, in particular, could appear especially problematic as future interventions cannot change past behavior. As noted, however, the claim in Study 3 is not that the experimental manipulation changed participants' past behavior, rather, we believe that it affected participants' construal of their own identities and behavior by affecting the recall of such behaviors. That is, the experimental manipulation changed the ease with which such behaviors were remembered and drawn to the forefront of participants' minds (Baumeister, Vohs \& Funder, 2007). Future studies along these lines should address these limitations by testing the impact of purity and groupbased appeals on actual behavior.

Furthermore, the samples in this work were limited to Indian participants due to initial comparison with the Clean India campaign and because purity, as a moral ideal, carries greater weigh amongst Indian populations. It is possible that the results described in this study could therefore not be 
directly relevant for populations from other geographic areas ${ }^{2}$. However, we believe that documentation of environmental behavior, values, attitudes, and norms within developing countries are of utmost importance. Not only because, as others have pointed out, the vast majority of psychology studies employ only American participants to generalize to larger populations (Henrich, Heine \& Norenzayan, 2010), but also because people residing in developing may be more vulnerable to climatic changes (Adger, Huq, Brown, Conway, \& Hulme, 2003).

\section{Conclusion}

Results from the current investigation increase our understanding of the means through which puritybased norms and appeals may impact environmental behavior. As previous research has shown, purity norms are an important value for many cultural and ideological groups and can increase environmental behavior in certain contexts. In this work, we suggest that in-group fusion is an important contextual factor that affects whether purity-based appeals either increase or, in some cases, decrease environmental behavior. As nations and communities begin to adapt to changing weather patterns, extreme climate events, and dwindling environmental resources, they will require effective appeals to promote sustainable and pro-environmental behavior. This work suggests that purity can be an effective norm to design an environmental appeal, particularly if it draws upon the group binding power of purity-based norms.

\footnotetext{
${ }^{2}$ It also remains an open question whether the findings from Studies 1 and 2 of the current manuscript would generalize to other sub-groups within India. For instance, do the nationalistic images from the Clean India Campaign speak as powerfully to Muslim Indian populations or to other populations which have been chronically underserved within the country?
} 


\section{Reference}

Atran, S., \& Norenzayan, A. (2004). Religion's evolutionary landscape: Counterintuition, commitment, compassion, communion. Behavioral and brain sciences, 27(6), 713-730.

Baumeister, R. F., Vohs, K. D., \& Funder, D. C. (2007). Psychology as the science of self-reports and finger movements: Whatever happened to actual behavior? Perspectives on Psychological Science, 2(4), 396-403.

Blei, D. M., Ng, A. Y., \& Jordan, M. I. (2003). Latent dirichlet allocation. Journal of machine Learning research, 3(Jan), 993-1022.

Boyer, P. (2008). Being human: Religion: bound to believe?. Nature, 455(7216), 1038-1039.

Bernbaum, E. (2006). Sacred mountains: Themes and teachings. Mountain Research and Development, 26(4), 304-309.

Dehghani, M., Johnson, K., Hoover, J., Sagi, E., Garten, J., Parmar, N. J., Vaisey, S., Iliev, R., \& Graham, J. (2016). Purity homophily in social networks. Journal of Experimental Psychology: General, 145(3), 366.

Douglas, M. (2003). Purity and danger: An analysis of concepts of pollution and taboo. Routledge.

Doron, A. (2016). Unclean, Unseen: Social Media, Civic Action and Urban Hygiene in India. South Asia: Journal of South Asian Studies, 39(4), 715-739.

Dunlap, R. E., Van Liere, K. D., Mertig, A. G., \& Jones, R. E. (2000). New trends in measuring environmental attitudes: measuring endorsement of the new ecological paradigm: a revised NEP scale. Journal of social issues, 56(3), 425-442. 
Running head: PURITY NORMS AND ENVIRONMENTAL BEHAVIOR

Eom, K., Kim, H. S., Sherman, D. K., \& Ishii, K. (2016). Cultural Variability in the Link Between Environmental Concern and Support for Environmental Action. Psychological science, 27(10), 1331-1339.

Feinberg, M., \& Willer, R. (2013). The moral roots of environmental attitudes. Psychological Science, 24(1), 56-62.

Gadgil, M., \& Vartak, V. D. (1976). The sacred groves of Western Ghats in India. Economic Botany, $30(2), 152-160$.

Garten, J., Hoover, J., Johnson, K. M., Boghrati, R., Iskiwitch, C., \& Dehghani, M. (2017). Dictionaries and distributions: Combining expert knowledge and large scale textual data content analysis. Behavior Research Methods, 1-18.

Gómez, A., Brooks, M. L., Buhrmester, M. D., Vázquez, A., Jetten, J., \& Swann Jr, W. B. (2011). On the nature of identity fusion: insights into the construct and a new measure. Journal of personality and social psychology, 100(5), 918.

Graham, J., Haidt, J., \& Nosek, B. A. (2009). Liberals and conservatives rely on different sets of moral foundations. Journal of personality and social psychology, 96, 1029.

Gupta, A., Coffey, D., \& Spears, D. (2016). Purity, pollution, and untouchability: challenges affecting the adoption, use, and sustainability of sanitation programmes in rural India. Sustainable Sanitation for All: Experiences, challenges, and innovations, 283.

Haidt, J. (2007). The new synthesis in moral psychology. Science, 316(5827), 998-1002.

Haidt, J., \& Graham, J. (2007). When morality opposes justice: Conservatives have moral intuitions that liberals may not recognize. Social Justice Research, 20(1), 98-116.

Haidt, J., Koller, S. H., \& Dias, M. G. (1993). Affect, culture, and morality, or is it wrong to eat your dog?. Journal of personality and social psychology, 65(4), 613. 
Running head: PURITY NORMS AND ENVIRONMENTAL BEHAVIOR

Hardin, G. (1968) The Tragedy of the Commons. Science, 162, 1243-1248.

Harris, M., Bose, N. K., Klass, M., Mencher, J. P., Oberg, K., Opler, M. K., Suttles, W., \& Vayda, A. P. (1966). The Cultural Ecology of India's Sacred Cattle [and Comments and Replies]. Current Anthropology, 7(1), 51-66.

Henrich, J., Heine, S., \& Norenzayan, A. (2010). The weirdest people in the world? Behavioral and Brain Sciences, 33(2-3), 61-83. doi:10.1017/S0140525X0999152X

Ice Bucket Challenge (n.d.). In Wikipedia. Retrieved September 10th, 2017, from https://en.wikipedia.org/wiki/Ice_Bucket_Challenge

Iliev, R., Dehghani, M., \& Sagi, E. (2015). Automated text analysis in psychology: Methods, applications, and future developments. Language and Cognition, 7(2), 265-290.

Jeffrey, R. (2015). Clean India! Symbols, Policies and Tensions. South Asia: Journal of South Asian Studies, 38(4), 807-819.

Karp, D. G. (1996). Values and their effect on pro-environmental behavior. Environment and behavior, 28(1), 111-133.

Lebbie, A. R., \& Guries, R. P. (1995). Ethnobotanical value and conservation of sacred groves of the Kpaa Mende in Sierra Leone. Economic Botany, 49(3), 297-308.

Neusner, J. (1975). The idea of purity in ancient Judaism. Journal of the American Academy of Religion, 43(1), 15-26.

Ross, M., McFarland, C., Conway, M., \& Zanna, M. P. (1983). Reciprocal relation between attitudes and behavior recall: Committing people to newly formed attitudes. Journal of Personality and Social Psychology, 45(2), 257. 
Running head: PURITY NORMS AND ENVIRONMENTAL BEHAVIOR

Rottman, J., \& Kelemen, D. (2012). Aliens behaving badly: Children's acquisition of novel purity-based morals. Cognition, 124(3), 356-360.

Rozin, P. (1996). Towards a psychology of food and eating: From motivation to module to model to marker, morality, meaning, and metaphor. Current Directions in Psychological Science, 5(1), 1824.

Sachdeva, S. (2016). Religious identity, beliefs, and views about climate change. Oxford Research Encyclopedia of Climate Science. http://dx.doi.org/10.1093/acrefore/9780190228620.013.335

Sachdeva, S. (2017). The influence of sacred beliefs in environmental risk perception and attitudes. Environment and Behavior, 49(5), 583-600. https://doi.org/10.1177/0013916516649413

Sachdeva, S., Jordan, J., \& Mazar, N. (2015). Green consumerism: moral motivations to a sustainable future. Current Opinion in Psychology, 6, 60-65.

Shweder, R., Much, N., Mahapatra, M., \& Park, L. (1997). Divinity and the "big three" explanations of suffering. Morality and health, 119, 119-169.

Sosis, R. (2011). Why sacred lands are not indivisible: The cognitive foundations of sacralising land. Journal of Terrorism Research, 2(1).

Sosis, R., \& Alcorta, C. (2003). Signaling, solidarity, and the sacred: The evolution of religious behavior. Evolutionary anthropology: issues, news, and reviews, 12(6), 264-274.

Swachh Bharat. (2014, October 20). Retrived from https://swachhbharat.mygov.in/

Swann Jr, W. B., Gómez, Á., Seyle, D. C., Morales, J., \& Huici, C. (2009). Identity fusion: the interplay of personal and social identities in extreme group behavior. Journal of personality and social psychology, 96(5), 995. 
Running head: PURITY NORMS AND ENVIRONMENTAL BEHAVIOR

Srull, T. K., \& Wyer, R. S. (1979). The role of category accessibility in the interpretation of information about persons: Some determinants and implications. Journal of Personality and Social psychology, 37(10), 1660.

Tversky, A., \& Kahneman, D. (1973). Availability: A heuristic for judging frequency and probability. Cognitive psychology, 5(2), 207-232.

Wolsko, C., Ariceaga, H., \& Seiden, J. (2016). Red, white, and blue enough to be green: Effects of moral framing on climate change attitudes and conservation behaviors. Journal of Experimental Social Psychology, 65, 7-19.

Zogmaister, C., Arcuri, L., Castelli, L., \& Smith, E. R. (2008). The impact of loyalty and equality on implicit in-group favoritism. Group Processes \& Intergroup Relations, 11(4), 493-512. 